Asian J Agric \& Biol. 2022(2).

$\mathrm{AJAB}$

DOI: $10.35495 / a j a b .2021 .02 .101$

Original Article

\title{
Diversity and mycorrhizal potential of arbuscular mycorrhizal fungi in two natural soils in the eastern region of Morocco
}

\author{
Wissame Chafai ${ }^{*}$, Soumaya El Gabardi ${ }^{2}$, Allal Douira ${ }^{2}$, Ahmed Khalid ${ }^{1}$ \\ ${ }^{1}$ Laboratory for the Improvement of Agricultural Production Biotechnology and Environment, Faculty of Sciences, \\ Mohammed I University, Oujda 60000, Moroccco \\ ${ }^{2}$ Laboratory of Plant Animal and Agro-Industry Productions, Botany, Biotechnology and Plant Protection Group, Faculty \\ of Sciences, Ibn Tofail University, Kenitra, Morocco
}

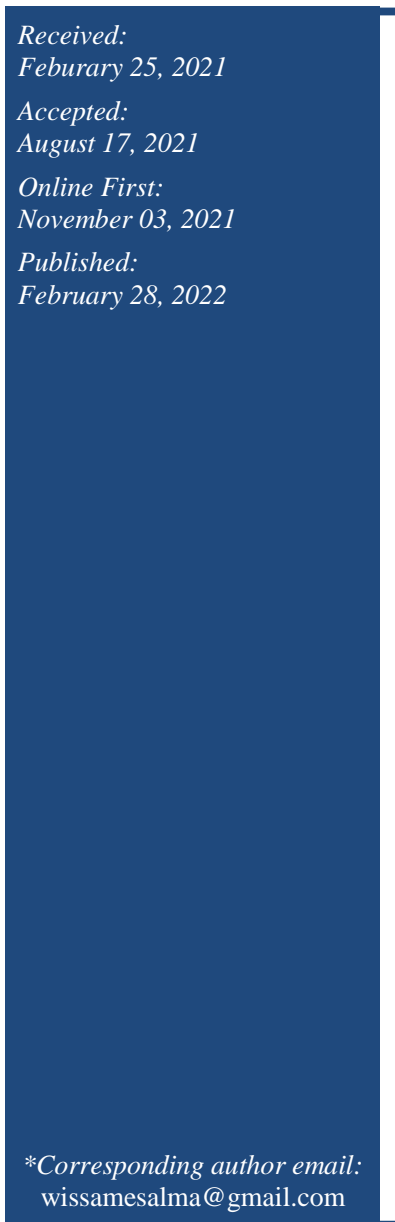

\begin{abstract}
This study aimed to assess the species composition and diversity of arbiscular mycorrhizal fungi (AMF) in two soil samples from two sites in the eastern region of Morocco; Guercif and Zaïo. The results showed that the spore densities of these sites extracted by wet sieving method were very high (279 and 386 spores/10 $\mathrm{g}$ of soil in Zaïo and Guercif sites, respectively). The provisional identification test of isolated AMF revealed the presence of 57 AMF species, belonging to five families (Glomeraceae, Gigasporaceae, Acaulosporaceae, Entrophosporaceae and Archaesporaceae). Glomus, Rhizophagus, Funneliformis, Endogone and Acaulospora were the dominant genera. In addition, mycorrhizal potential of both soils was assessed using the "Most Probable Number" (MPN) method. The results revealed that the number of mycorrhizal propagules in Guercif soil were higher than that in Zaïo soil. It was also shown that the frequency and the intensity of root mycorrhization of leeks transplanted in Guercif soil were higher (90\% and $74 \%$, respectively) compared to those transplanted in Zaïo soil (56\% and 31\%, respectively). These results showed that both soils are generally rich in mycorrhizal fungal propagules and have great mycorrhizogenic power, so it would be interesting to isolate and purify fungal strains and to select those that perform well for a given parameter.
\end{abstract}

Keywords: Arbuscular mycorrhizal fungi, Diversity, Most probable number, Frequency and intensity of mycorrhization

\section{How to cite this:}

Chafai W, EL Gabardi S, Douira A and Khalid A, 2022. Diversity and mycorrhizal potential of arbuscular mycorrhizal fungi in two natural soils in the eastern region of Morocco. Asian J. Agric. Biol. 2022(2): 202102101. DOI: https://doi.org/10.35495/ajab.2021.02.101

This is an Open Access article distributed under the terms of the Creative Commons Attribution 3.0 License. (https://creativecommons.org/licenses/by/3.0), which permits unrestricted use, distribution, and reproduction in any medium, provided the original work is properly cited.

\section{Introduction}

Currently, agriculture faces several major challenges, one of which is the rapid growth of the human population, estimated at 8.9 billion in 2050 (Damon, 2003). Overcoming the dietary needs of the growing population requires an increase in crop yield, and a move towards intensive agricultural production that is both productive and environmentally sustainable with limited or no use of synthetic fertilizers and chemicals. The alternative of a more productive agriculture that is less dependent on external inputs, such as fertilizers, is 
not possible without better management of interactions between microorganisms and the plants within the agrosystems (Plenchette et al., 2005; Adesemoye and Kloepper, 2009). One of these interactions is the mycorrhizae, which are symbiotic associations between fungi and plants that give plants many benefits.

AMF, which are considered bio-fertilizers, are a naturel constituent of soil in most ecosystems and can be found in almost all soils in both dry and temperate zones (Pamiske, 2008). The benefits of AMF in forest and agricultural ecosystems are widely recognized. In addition to its contribution to soil aggregation and structural stability (Rillig et al., 2002), AMF were shown to improve mineral nutrition by enhancing the plants ability to uptake mineral nutrients, (Bencherif et al., 2015), especially phosphate (Sally et al., 2003). Moreover, AMF was shown to confer plants protection against abiotic stresses (such as drought, salinity, heavy metals and changes in temperature) (Begum et al., 2019), as well as against pathogens, pests, and parasitic plants (Jung et al., 2012).

Despite the interest and importance of AMF, the use of these microorganisms in agriculture is still limited, mainly due to the incompatibility between the strains introduced and local edaphic characteristics (Duponnois et al., 2013), causing disappearance of fungi from the injected inoculum. Thus, it is crucial to select native strains, which can adapt to the constraints of the environment.

The aim of our study is to evaluate the diversity and abundance of AMF species in two soils, from two sites in the east of Morocco (Guercif and Zaïo), which would interesting to exploit them in the development of a composite endomycorhizal inoculum. The choice of these two sites was based on their potential agricultural production, especially the plain of Guercif which was recently exploited, in contrast to the plain of sabra (Zaïo) which has been cultivated for about 50 years. We also aimed, in the present study, to evaluate the mycorrhizogenic potential in order to enhance their native AMF species. Mycorrhizogenic potential was assessed by the "Most Probable Number" or MPN method (Alexander, 1965; Porter, 1979; Wilson and Trinick, 1982; Gianinazzi-Pearson et al., 1985). The MPN bioassay estimate of the number of infective propagules per weight of the tested soil, which reflect the ability of a soil to initiate the formation of mycorrhizal associations from a quantity of inoculum present in the soil as propagules (Plenchette et al., 1989).

\section{Material and Methods}

\section{Soil sample}

In this study, natural soil samples were collected from two sites in eastern Morocco with two different agro-pedological conditions: Guercif and Zaïo whose coordinates are $\left(33^{\circ} 58^{\prime} 45^{\prime \prime} \mathrm{N}, 3^{\circ} 15^{\prime} 41^{\prime \prime} \mathrm{W}\right.$, Altitude: $688 \mathrm{~m}$, and $34^{\circ} 54^{\prime} 14^{\prime \prime} \mathrm{N}, 2^{\circ} 48^{\prime} 49^{\prime \prime} \mathrm{W}$, Altitude: $138 \mathrm{~m}$, respectively).

Four random samples were taken from each site at a depth ranging from 0 to $30 \mathrm{~cm}$; and a composite soil sample was produced for each site.

Physicochemical characteristics of soils were determined by two laboratories AGRILABO in Fes and LACQ in Meknes (Morocco).

\section{Spore isolation}

Isolation of AMF spores was carried out by wet sieving using the technique of Gerdemann and Nicolson (Gerdemann and Nicolson, 1963), followed by centrifugation on a sucrose gradient using the method of Giovannetti et al (Giovannetti et al., 1991). $10 \mathrm{~g}$ of each soil sample was mixed with $500 \mathrm{ml}$ of tap water in a beaker and stirred vigorously. The soil suspension was then passed through a sieve column made up of two sieves of $500 \mu \mathrm{m}$ and $50 \mu \mathrm{m}$ mesh, under a water jet. The $50 \mu \mathrm{m}$ sieve was collected in distilled water. The resulting spore suspension was then divided into five tubes and centrifuged for $5 \mathrm{~min}$ at $9000 \mathrm{rev} / \mathrm{min}$. after discarding the supernatant, a viscosity gradient was created by adding $15 \mathrm{ml}$ of sucrose solution at $60 \%$ to each centrifuge tube. The mixture was then rapidly stirred and centrifuged for 4 $\mathrm{min}$ at $3000 \mathrm{rev} / \mathrm{min}$. The supernatant containing the spores was filtered and rinsed through a $50 \mu \mathrm{m}$ sieve with distilled water to remove sucrose. The spores were then recovered with distilled water in a petri dish.

Isolated spores were quantified by direct counting under a binocular magnifying glass to estimate the number of spores in $10 \mathrm{~g}$ of soil of each sample (spore density). Five repetitions were performed for each extraction.

Appearance frequency of species (A.F.S \%) designates the percentage of a morphotype relative to total number of species. A.F.S $\%=(\mathrm{ns} / \mathrm{nT}) * 100$, where ns is the isolated spores number of the species $\mathrm{X}$ and $\mathrm{nT}$ is the total spores number.

Appearance frequency of genus (A.F.G): designates the percentage of a total spore species of one genus relative to the total spores .A.F.G $\%=(\mathrm{nG} / \mathrm{nT})$ $* 100$, where $\mathrm{nG}$ is the number of spores of the genus $\mathrm{X}$ and $\mathrm{nT}$ is the total spores number. 


\section{Spore identification}

The extracted spores of the AMF were observed between a slide and a cover slip under the microscope and then photographed. Taxonomic identification of spores down to the species level was based on the size, color, shape, wall structure and hyphal attachments of the spore. The identification of our spores has been carried out using species descriptions provided by the International Collection of Cultures of Arbuscular Vesicular Mycorrhizal Mushrooms (INVAM, 2017) and other appropriate references (Walker and Mize, 1982; Schenk and Perez, 1987; Schenk and Perez, 1990; Morton and Benny, 1990; Morton and Bentivenga, 1994).

\section{Estimation of the mycorrhizogenic potential of the studied soils}

Mycorrhizal potential of soils was determined by the "Most Probable Number" (MPN) bioassay, which quantify all infective propagules of AMF in a specific soil (Plenchette et al., 1989).

The soil samples were dried at room temperature, then sieved through a $2 \mathrm{~mm}$ mesh sieve.

The resulting soils were diluted with sterilized sand (autoclaved at $120{ }^{\circ} \mathrm{C}$ for $30 \mathrm{~min}$ ).

Five dilutions $(1 / 1,1 / 4,1 / 16,1 / 64,1 / 256)$ were made with five replicates.

Two weeks old leek (Allium porrum) seedlings were transplanted into $7 / 7 / 8 \mathrm{~cm}$ plastic pots, previously filled with the substrates from the dilutions (100 $\mathrm{g} /$ pot). All the pots were placed in a greenhouse afterward. After 10 weeks of cultivation, the plants were dug up and the root system of each plant was recovered, washed and stained to reveal the presence of mycorrhizal structures, according to the technique described by Phillips and Hayman (Phillips and Hayman, 1970).

Mycorrhizal structures were observed under the microscope. Each root system showing at least one point of infection (penetration of a hypha in the root) was considered to be mycorrhizal.

The MPN of propagules was calculated by the following formula:

$\log$ MPN $=\left(\mathrm{X}^{*} \log \mathrm{a}\right)-\mathrm{K}_{(\mathrm{y}, \mathrm{S})}$, where $" \mathrm{X}^{\prime}$ is the average number of positives per repetition, "a" is the dilution factor and " $\mathrm{K}$ " was determined in the Fisher and Yates table (Fisher and Yates, 1970) as a function of "Y" (The average number of negatives per repeat) and $S$.

$\mathrm{S}$ is the dilution number
Cochran defines a formula approximating (SE), the standard deviation of $\log$ MPN whose distribution is close to the normal distribution (Cochran, 1950):

$$
\mathrm{SE}=0.55 \sqrt{\left(\frac{\log a}{\mathrm{n}}\right)}
$$

Where "a" is the dilution factor and " $\mathrm{n}$ " is the number of repetitions.

The $95 \%$ confidence interval is obtained as follows:

$$
\mathrm{CI}(95 \%)=\log (\mathrm{MPN}) \pm 2 \mathrm{SE}
$$

To find the lower and upper limits, simply divide and multiply the estimated MPN value by Antilog (2SE), respectively.

\section{Frequency of mycorrhization and intensity of root colonization}

Mycorrhization was estimated according to the method described by Trouvelot et al. (Trouvelot et al., 1986), allowing the calculation of the two parameters below:

Mycorrhization frequency $(\mathrm{F}): \mathrm{F} \%=((\mathrm{N}-\mathrm{No}) / \mathrm{N})$ $\mathrm{x} 100$, where $\mathrm{N}$ is the number of fragments observed and No is the number of fragments with no evidence of mycorrhization.

Colonization intensity (M\%), which expresses the portion of the colonized cortex in relation to the entire root system: $M \%=[(95 \times n 5)+(70 \times n 4)+(30$ $\mathrm{x} \mathrm{n} 3)+(5 \times \mathrm{n} 2+\mathrm{n} 1)] / \mathrm{N}$, where "N" : number of fragments observed and $n 5, n 4, n 3, n 2$ and $n 1$ are the numbers of fragments noted $5,4,3,2$ and 1 respectively; Class 5 : over $91 \%$, class 4 : from $51 \%$ to $91 \%$, class 3 : from 11 to $50 \%$, class 2 : less than $10 \%$, class $1: 1 \%$ and class $0:$ no mycorrhization.

\section{Statistical analysis}

All data were analysed using the analysis of variance to a single criterion of classification (ANOVA) or Student t-test when appropriate. Data analysis was performed using SPSS version 20 software for Windows. Values of $p<0.05$ were considered statistically significant.

\section{Results}

The physicochemical analyses of the two soils, showed that Zaio soil is a limestone soil, and the Guercif soil have a heavy clay-silt texture. Both 
Guercif soil and Zaïo soil were alkaline $(\mathrm{pH} ; 8.3$ and 8.1 respectively) and contained very little organic matter $(1.04 \%$ and $2.34 \%$, respectively), but were rich in potassium (618 ppm and $910 \mathrm{ppm}$, respectively) (Table 1).

Guercif soil was low in phosphorus (7.27 ppm) and nitrogen (1.33 mg/100 g soil), however, Zaïo soil was high in phosphorus (74.9 ppm) and medium in nitrogen $(170 \mathrm{mg} / 100 \mathrm{~g}$ soil $)$.

Table-1: Physicochemical characteristics of Guercif soil and Zaïo soil

\begin{tabular}{|c|c|c|}
\hline Site & $\begin{array}{c}\text { Guerc } \\
\text { if }\end{array}$ & Zaïo \\
\hline Clay $\%$ & 36.25 & 56 \\
\hline Limes\% & 34.69 & 30 \\
\hline Sands $\%$ & 29.07 & 14 \\
\hline $\mathrm{pH}$ & 8.30 & 8.1 \\
\hline E.C à $25 \mathrm{C}^{\circ}(\mathrm{mmhos} / \mathrm{cm})($ extract $1 / 5)$ & 0.189 & 0.546 \\
\hline Sodium $(\mathrm{mg} / \mathrm{kg})$ & 64.00 & 412 \\
\hline Nitrogen $\mathrm{mg} / 100 \mathrm{~g}$ of soil & 1.33 & 170 \\
\hline Organic matter (\%) & 1.04 & 2.34 \\
\hline Total limestone $(\%)$ & traces & 12.2 \\
\hline Phosphorus(ppm: part-per-million) & 7.27 & 74.9 \\
\hline Potassium (ppm) & 618.80 & 910 \\
\hline Calcium (ppm) & $\begin{array}{c}2658.0 \\
0\end{array}$ & 13700 \\
\hline Magnesium (ppm) & 185.00 & 1289 \\
\hline
\end{tabular}

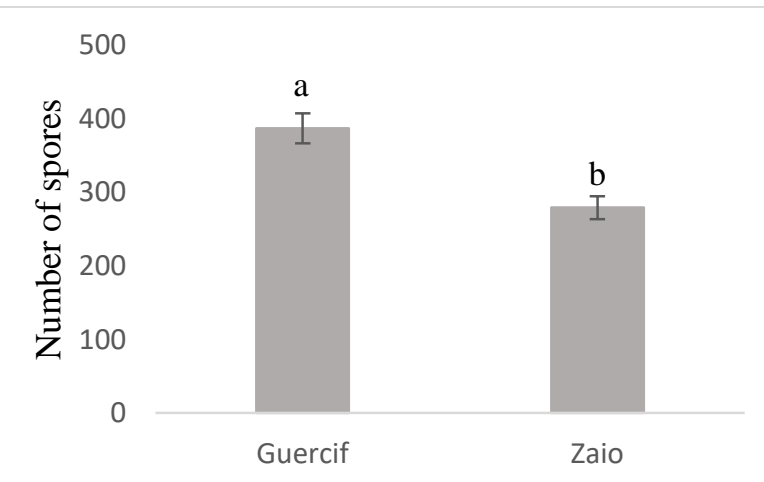

Figure-1: Spore abundance in Guercif and Zaïo soil. Bars with the different letter were signifcantly different $(P<0.05)$
Spore density and richness at the two study sites The abundance of AMF spores found in the two study sites was 279 per $10 \mathrm{~g}$ of Zaïo soil and 386 per $10 \mathrm{~g}$ of Guercif soil; they show a significant difference between soils (Figure 1).

Preliminary identifications (Table 2 and Figure 2) showed that the spores isolated from the two sites belonged to 57 species: Glomus macrocarpum; Glomus microcarpum; Glomus glomerulatum; Rhizophagus intraradices; Claroideoglomus lamellosum; Rhizophagus fasciculatum; Glomus rubiforme; Pacispora chimonobambusae; Rhizophagus clarus; Glomus clavisporum ; Endogone versiformis ; Funneliformis mosseae; Claroideoglomus claroideum ; C. etunicatum; Glomus albidum; Funneliformis constrictum; Glomus deserticola; Glomus aggregatum; Glomus viscosum ; Funneliformis geosporum; Glomus spinuliferum; $G$. boreale; G. minutum; Glomus sp 1; Glomus sp 2; Glomus sp 3 ;Glomus sp 4 ; Glomus sp 5 ; Glomus sp 6; Glomus sp 7; Glomus sp 8; Glomus sp 9; Acaulospora leavis; Acaulospora colombiana; Acaulospora capsicula; Acaulospora herrerae; A. scrobiculata; A. mellea; A. gedanensis ; A. foveata; A. morrowiae; Acaulospora brasiliensis; Acaulospora spinosa; Acaulospora sp 1; Acaulospora sp 2; Acaulospora sp 3 ; Acaulospora sp 4; Acaulospora sp 5; Scutellospora coralloidea; Dentisculata nigra; Scutellospora calospora ; Claroideoglomu drummondii; Pacispora chimonobambusae; Gigaspora margarita; Glomus coremioides; Entrophospora infrequens and Archaeospora trappei.

All these species found in the two sites belong to eleven genera (Glomus, Funneliformis, Rhizophagus, Acaulospora, Entrophospora, Dentiscutata, Scutellospora, Claroideoglomus, Archaespora, Gigaspora, Pacispora, and Endogone), five families (Glomeraceae, Gigasporaceae, Acaulosporaceae, Entrophosporaceae and Archaesporaceae) and three Orders (Diversisporales, Glomerales and Archaesporales). 
Wissame Chafai et al.

Table-2: Characteristics of all AMFs isolated from the soils of the two sites (Guercif and Zaïo)

\begin{tabular}{|c|c|c|c|c|c|c|c|}
\hline No. & Species & form & color & $\begin{array}{c}\text { spore size } \\
(\mu \mathrm{m})\end{array}$ & $\begin{array}{c}\begin{array}{c}\text { Wall size } \\
(\mu \mathrm{m})\end{array} \\
\end{array}$ & $\begin{array}{c}\text { Length of } \\
\text { the hypha }(\mu \mathrm{m})\end{array}$ & $\begin{array}{c}\text { Surface of } \\
\text { the spore }\end{array}$ \\
\hline 1 & Glomus macrocarpum & Globular & Orange brown & 145 & 1,3 & - & Smooth \\
\hline 2 & Glomus microcarpum & Globular & Brown & 84 & 1 & - & Grainy \\
\hline 3 & Glomus glomerulatum & Globular & Brown & 120 & 2,5 & - & Smooth \\
\hline 4 & Rhizophagus intraradices & Globular & Hyaline & 157 & 6,7 & - & Smooth \\
\hline 5 & Claroideoglomus lamellosum & Globular & Orange & 229 & 4,5 & 81 & Smooth \\
\hline 6 & Glomus rubiforme & Globular & Yellow & 174 & 2 & 48 & Smooth \\
\hline 7 & Pacispora chimonobambusae & Sub-globular & Pale yellow & 84 & 2 & - & Grainy \\
\hline 8 & Rhizophagus clarus & Globular & Brown & 193 & 3 & 174 & Smooth \\
\hline 9 & Glomus clavisporum & Globular & Brown & 96 & 1 & - & Grainy \\
\hline 10 & Endogone versiformis & Globular & Brown & 133 & 1,5 & - & Grainy \\
\hline 11 & Funneliformis mosseae & Globular & Yellow & 84 & 1,3 & - & Smooth \\
\hline 12 & Claroideoglomus claroideum & Globular & Pale yellow & 84 & 1,5 & - & Smooth \\
\hline 13 & Claroideoglomus etunicatum & Globular & orange & 104 & 2 & - & Smooth \\
\hline 14 & Glomus albidum & Globular & Brown & 133 & 3,3 & - & Smooth \\
\hline 15 & Funneliformis constrictum & Globular & Almost black & 72 & 1 & - & Smooth \\
\hline 16 & Glomus deserticola & Globular & Reddish brown & 96 & 1,3 & - & Smooth \\
\hline 17 & Glomus viscosum & sub-globular & Pale yellow & 101 & 2,7 & - & Smooth \\
\hline 18 & Funneliformis geosporum & Globular & orange brown & 122 & 8 & - & Smooth \\
\hline 19 & Glomus spinuliferum & Globular & Yellow & 72 & 2,5 & - & Smooth \\
\hline 20 & Glomus boreale & Globular & Black & 108 & 2,3 & 30 & Smooth \\
\hline 21 & Glomus minutum & Globular & hyaline & 169 & 4,2 & 37 & Smooth \\
\hline 22 & Pacispora chimonobambusae & Globular & Pale yellow & 133 & 3 & - & Grainy \\
\hline 23 & Glomus sp 1 & Globular & Orange brown & 84 & 2,5 & - & Smooth \\
\hline 24 & Glomиs sp 3 & Gglobular & Brown & 104 & 1,8 & - & Smooth \\
\hline 25 & Rhizophagus intraradices & Globular & hyaline & 120 & 7 & 71 & Smooth \\
\hline 26 & Glomus sp 4 & Globular & Brown to black & 72 & 1,6 & - & Smooth \\
\hline 27 & Acaulospora capsicula & Globular & Brown & 181 & 6 & - & Smooth \\
\hline 28 & Glomus sp 7 & Oval & Brown & 96 & 1,3 & - & Grainy \\
\hline 29 & Glomus sp 8 & Oval & $\begin{array}{c}\text { Dark brown to } \\
\text { black }\end{array}$ & 96 & 2,2 & - & Smooth \\
\hline 30 & Glomus versiforme & Globular & Brown & 157 & 3 & - & Grainy \\
\hline 31 & Acaulospora laevis & Globular & Pale brown & 82 & 3,3 & - & Smooth \\
\hline 32 & Glomus sp 5 & Globular & Orange & 72 & 1,7 & - & Smooth \\
\hline 33 & Acaulospora colombiana & Globular & Yellow & 169 & 2,8 & - & Smooth \\
\hline 34 & Glomus sp 9 & Oval & Brown & 101 & 1,5 & - & Smooth \\
\hline 35 & Rhizophagus intraradices & Globular & Yellow & 58 & 0,8 & 23 & Smooth \\
\hline 36 & Glomus sp 6 & Globular & Yellow & 140 & 4 & - & Smooth \\
\hline 37 & Acaulospora herrerae & Globular & Yellow & 205 & 7 & - & Smooth \\
\hline 38 & Acaulospora scrobiculata & Globular & Sub-hyaline & 82 & 0,8 & - & Grainy \\
\hline 39 & Acaulospora mellea & Globular & Brown-orange & 120 & 1 & 109 & Smooth \\
\hline
\end{tabular}


Wissame Chafai et al.

\begin{tabular}{|c|c|c|c|c|c|c|c|}
\hline 40 & Acaulospora gedanensis & Globular & hyaline & 72 & 3 & 30 & Smooth \\
\hline 41 & Acaulospora foveata & $\begin{array}{c}\text { Sub- } \\
\text { globular }\end{array}$ & Orange & 96 & 3 & 31 & Smooth \\
\hline 42 & Acaulospora morrowiae & Globular & Yellow & 108 & 6,4 & - & Grainy \\
\hline 43 & Acaulospora brasiliensis & Oval & Yellow & 84 & 1,6 & 52 & Rough \\
\hline 44 & Acaulospora spinosa & Globular & Yellow-brown & 133 & 2,7 & 37 & Thorny \\
\hline 45 & Acaulospora sp 1 & Globular & Grown & 133 & 2,5 & 75 & Smooth \\
\hline 46 & Rhizophagus intraradices & Globular & Hyaline & 96 & 1,5 & 99 & Smooth \\
\hline 47 & Rhizophagus fasciculatum & Globular & Brown & 84 & 3,3 & - & Smooth \\
\hline 48 & Acaulospora sp 2 & Globular & Hyaline & 120 & 0,8 & 22 & Smooth \\
\hline 49 & Funneliformis mosseae & Globular & Yellow & 91 & 2,8 & - & Smooth \\
\hline 50 & Acaulospora sp 5 & Globular & Hyaline & 84 & 1,5 & 18 & Smooth \\
\hline 51 & Scutellospora coralloidea & Globular & Yellow & 174 & 3,5 & - & Smooth \\
\hline 52 & Dentiscutata nigra & Globular & Black & 133 & 2,6 & - & Smooth \\
\hline 53 & Scutellospora calospora & Globular & Pastel yellow & 165 & 1.5 & - & Smooth \\
\hline 54 & $\begin{array}{l}\text { Claroideoglomus } \\
\text { drummondii }\end{array}$ & Globular & Hyaline & 96 & 1,7 & - & Smooth \\
\hline 55 & Acaulospora sp 3 & Globular & Yellow & 72 & 4 & - & Smooth \\
\hline 56 & Gigaspora margarita & Globular & Yellow & 84 & 1,5 & - & Smooth \\
\hline 57 & Glomus spinuliferum & Globular & Orange & 229 & 12 & & Smooth \\
\hline 58 & Funneliformisgeosporum & sub-globular & Brown-orange & 84 & 1,7 & 36 & Smooth \\
\hline 59 & Glomus coremioides & Globular & Brown & 72 & 2,5 & - & Grainy \\
\hline 60 & Entrophospora infrequens & Globular & Brown-orange & 241 & 4 & - & Smooth \\
\hline 61 & Archaeospora trappei & Globular & Hyaline & 91 & 1,5 & - & Smooth \\
\hline 62 & Acaulospora sp 4 & Globular & Yellow & 70 & 1,2 & - & Smooth \\
\hline 63 & Glomиs multicaule & Globular & Yellow & 120 & 1 & 108 & Smooth \\
\hline 64 & Glomиs macrocarpum & Globular & Yellow-brown & 84 & 11 & - & Smooth \\
\hline 65 & Glomus microcarpum & Globular & Brown & 169 & 4,5 & 61 & Smooth \\
\hline 66 & Glomus sp 2 & Globular & Hyaline & 193 & 2,2 & 48 & Smooth \\
\hline 67 & Acaulospora laevis & Globular & Yellow & 104 & 2,5 & - & Smooth \\
\hline 68 & Glomus aggregatum & Globular & Brown & 96 & 1,5 & - & Smooth \\
\hline 69 & Acaulospora colombiana & Ellipsoid & Orange & 84 & 1,4 & - & Smooth \\
\hline 70 & Acaulospora scorbiculata & Globular & Light brown & 96 & 3 & - & Grainy \\
\hline
\end{tabular}

Species of endomycorrhizal fungi such as Glomus sp9, Acaulospora scorbiculata, Acaulispora mellea, A. gedeanensis, A. foveata, Glomus viscosum, Archaeispora trappei, Acaulospora sp4, Glomus boreale, Glomus sp4, Glomus sp5, Glomus sp6, Glomus sp7, Glomus sp8, Acaulospora sp3, Glomus minitum, Acaulospora brasilliensis, Acaulospora spinosa, Acaulospora marrowiae, Acaulospora sp5 and Glomus aggregatum, were found in Guercif soil, but not in Zaïo soil. On the other hand, Acaulospora colombiana, Claoideoglomus lamellosum, Glomus sp2, Claroideoglomus drumondii, Glomus rubiforme, Acaulospora sp1, Scutellospora coralloidea, Glomus multicaule, Claroideoglomus claroideum, Glomus sp1, Glomus albidum, G. coremiö̈des, Acaulospora sp2, Acaulospora capsicula, Acaulospora herrerae, were present in Zaïo soil, but not in Guercif soil (Table 3 and Figures 3 and 4). 
Wissame Chafai et al.

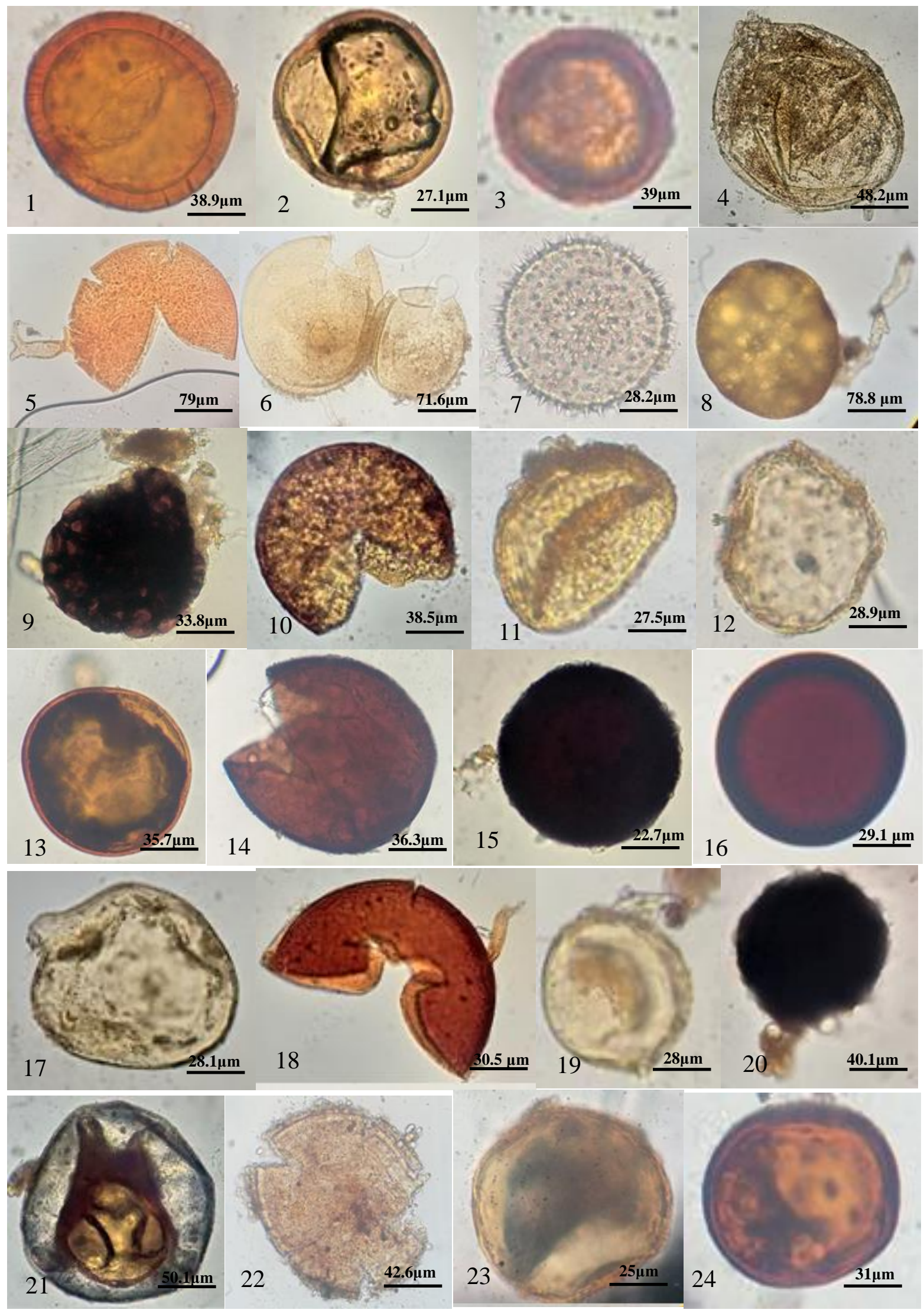

(1) Asian J Agric \& Biol. 2022(2). 
Wissame Chafai et al.

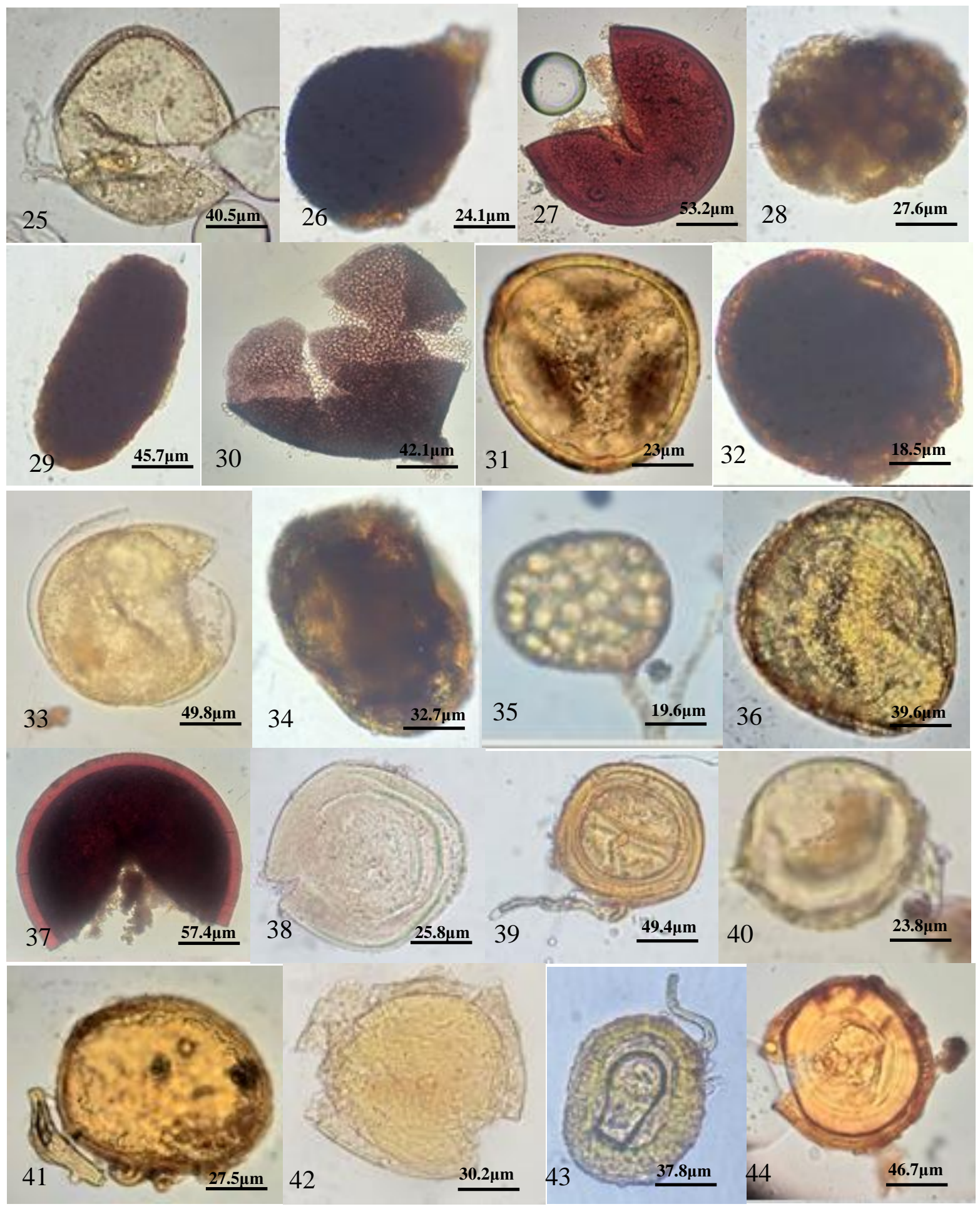


Wissame Chafai et al.

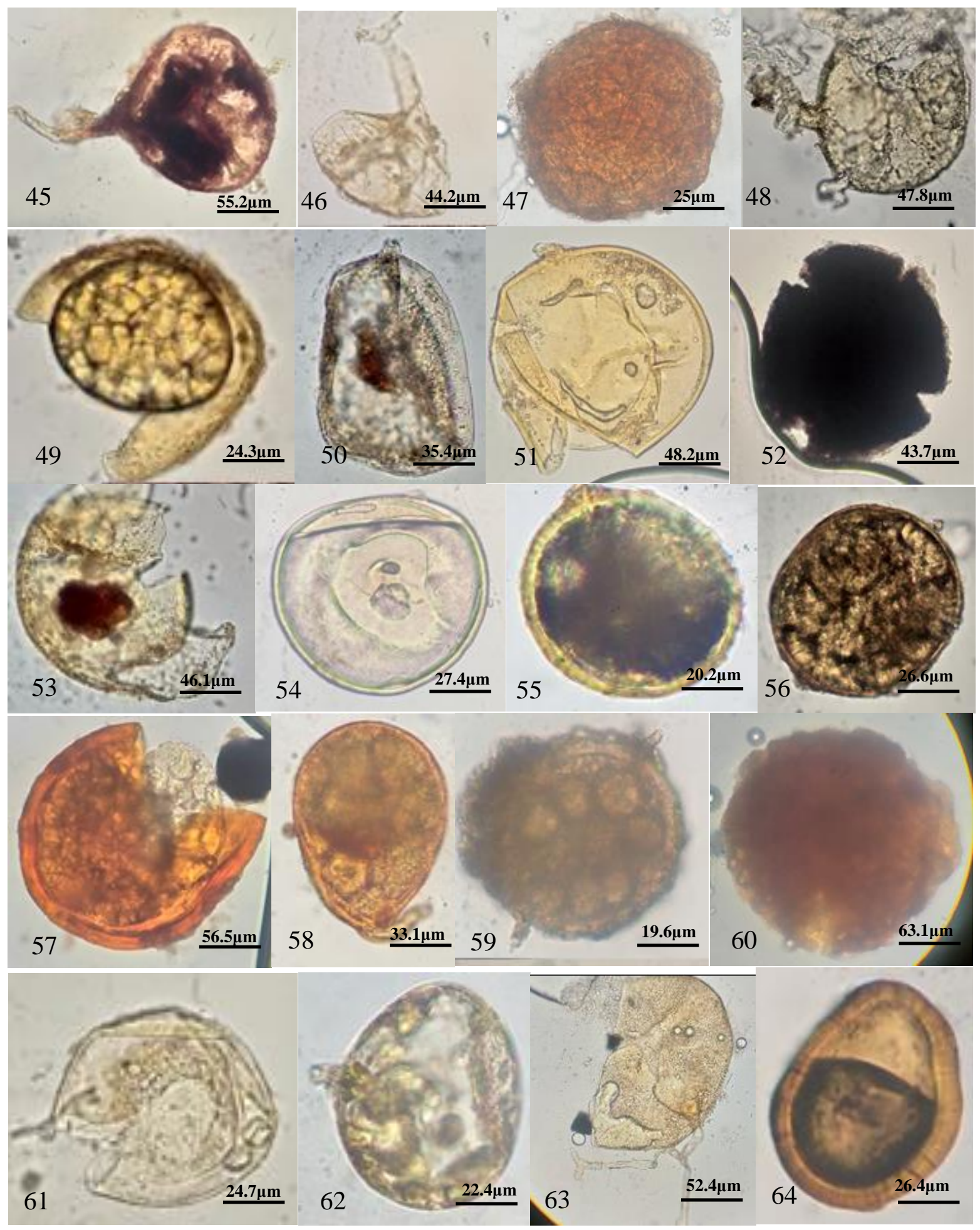

(1) Asian J Agric \& Biol. 2022(2). 


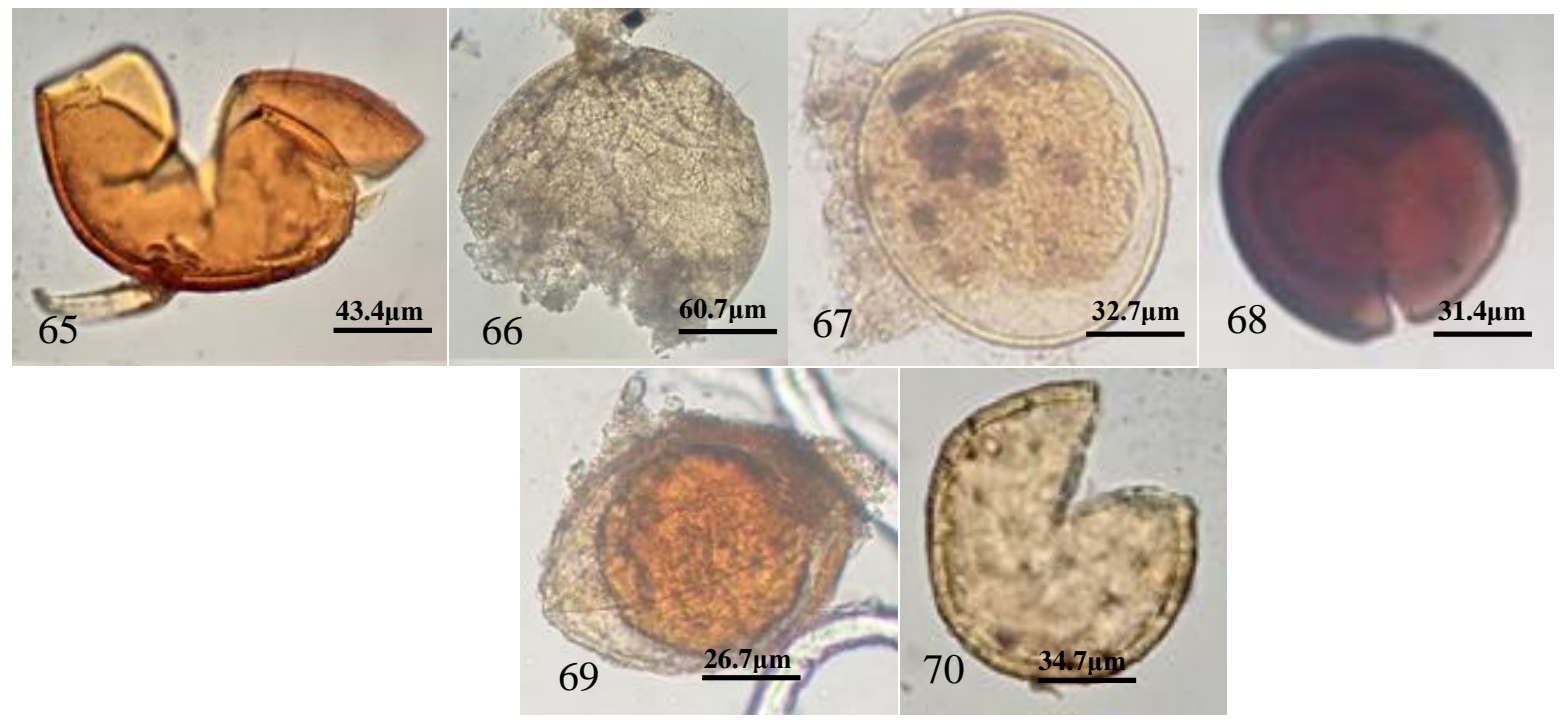

Figure-2: Photos of endomycorrhizal fungi species found in the soil of two sites in eastern Morocco: Guercif and Zaïo.

Glomus macrocarpum (1); Glomus microcarpum (2); Glomus glomerulatum (3) ; Rhizophagus intraradices (4); Claroideoglomus lamellosum (5); Glomus rubiforme (6); Pacsispora chimonobambusae (7); Rhizophagus clarus(8); Glomus clavisporum (9); Endogone versiforme (10) ; Funneliformis mosseae (11); Claroideoglomus claroideum (12); Claroideoglomus etunicatum (13); Glomus albidum (14); Funneliformis constrictum (15); Glomus deserticola (16) ; Glomus viscosum (17) ; Funneliformis geosporum (18) ; Glomus spinuliferum (19) ; Glomus boreale (20); Glomus minutum (21); Pacispora chimonobambusae (22) ; Glomus sp 1(23); Glomus sp3 (24); Rhizophagus intraradices (25); Glomus sp4 (26); Acaulospora capsicula (27); Glomus sp7 (28); Glomus sp8 (29); Glomus versiforme (30); Acaulospora leavis (31); Glomus sp5 (32); Acaulospora colombiana (33); Glomus sp9 (34); Rhizophagus intraradices (35); Glomus sp6 (36); Acaulospora herrerae (37) ; Acaulospora scrobiculata (38); Acaulospora mellea (39) ; Acaulospora gedanensis (40) ; Acaulospora foveata (41); Acaulospora morrowiae (42); Acaulospora brasiliensis (43); Acaulospora spinosa (44); Acaulospora sp1 (45); Rhizophagus intraradices (46) ; Rhizophagus fasciculatum (47); Acaulospora sp2 (48); Funneliformis mosseae (49); Acaulospora sp5 (50); Scutellospora coralloidea (51); Dentiscutata nigra (52) ; Scutellospora calospora (53) ; Claroideoglomus drummondii (54) ; Acaulospora sp3 (55); Gigaspora margarita (56); Glomus spinuliferum (57); Funneliformis geosporum (58); Glomus coremioides (59) ; Entrophospora infrequens (60) ; Archaeospora trappei (61) ; Acaulospora sp4 (62) ; Glomus multicaule (63); Glomus macrocarpum (64); Glomus microcarpum (65); Glomus sp2 (66) ; Acaulospora laevis (67) ; Glomus aggregatum (68); Acaulospora colombiana (69) ; Acaulospora scorbiculata (70) 
Wissame Chafai et al.

Table-3: Appearance of AMF species in soils at study sites (+): Present and (-): Absent

\begin{tabular}{|c|c|c|}
\hline Species & Site Guercif & Site Zaïo \\
\hline Glomus deserticola & + & + \\
\hline Scutellospora coralloidea & - & + \\
\hline Claroideoglomus drummondii & - & + \\
\hline Rhizophagus intraradices & + & + \\
\hline Scutelospora nigra & + & + \\
\hline Glomus macrocarpum & + & + \\
\hline Glomus aggregatum & + & - \\
\hline Acaulospora scorbiculata & + & - \\
\hline Claroideglomus lamellosum & - & + \\
\hline Glomus glomerulatum & + & + \\
\hline Glomus rubiforme & - & + \\
\hline Acaulopora colombiana & - & + \\
\hline Acaulospora mellea & + & - \\
\hline Acaulospora gedanensis & + & - \\
\hline Acaulospora foveata & + & - \\
\hline Glomus viscosum & + & - \\
\hline Glomus multicaule & - & + \\
\hline Rhizophagus clarus & + & + \\
\hline Funneliformis geosporum & + & + \\
\hline Archaeospora trappei & + & - \\
\hline Acaulopora capsicula & - & + \\
\hline Glomus spineliforum & + & + \\
\hline Glomus boreale & + & - \\
\hline Rhizophagus fasciculatum & + & + \\
\hline Funneliformis mosseae & + & + \\
\hline Glomus $\mathrm{sp} 7$ & + & - \\
\hline Glomus sp8 & + & - \\
\hline Claroideoglomus claroideum & - & + \\
\hline Endogone versiformis & + & + \\
\hline Glomus sp3 & + & + \\
\hline Glomus sp2 & - & + \\
\hline Acaulospora $\mathrm{sp} 4$ & + & - \\
\hline Glomus sp9 & + & - \\
\hline Glomus sp4 & + & - \\
\hline Glomus sp5 & + & - \\
\hline Claroideoglomus etunicatum & + & + \\
\hline Glomus albidum & - & + \\
\hline Glomus microcarpum & + & + \\
\hline Acaulospora sp1 & - & + \\
\hline Acaulospora $\mathrm{sp} 3$ & + & - \\
\hline Glomus coremioides & - & + \\
\hline Glomus sp6 & + & - \\
\hline Glomus minitum & + & - \\
\hline Acaulospora sp5 & + & - \\
\hline Acaulospora laevis & + & + \\
\hline Acaulospora brasilliensis & + & - \\
\hline Acaulospora spinosa & + & - \\
\hline Glomus clavisporum & + & + \\
\hline Acaulospora herrerae & - & + \\
\hline Funneliformis constructum & + & + \\
\hline Pacispora chimonbanbusae & + & + \\
\hline Glomus sp1 & - & + \\
\hline Acaulospora sp2 & - & + \\
\hline Gigaspora margarita & + & + \\
\hline Acaulospora marrowiae & + & - \\
\hline Entrophospora infrequens & + & + \\
\hline Scutelospora calospora & + & + \\
\hline
\end{tabular}

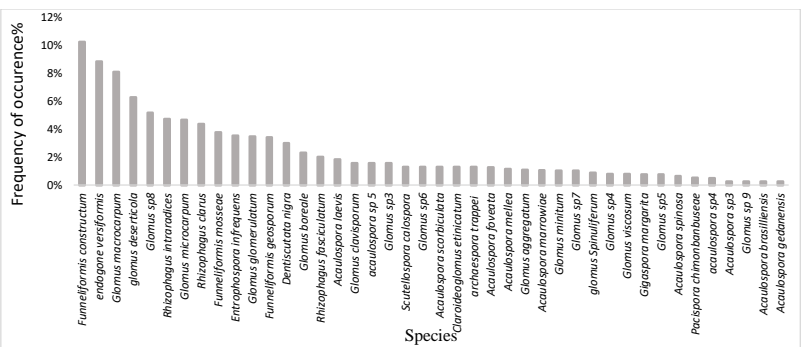

Figure-3: Frequency of occurrence of mycorrhizal species in Guercif soil

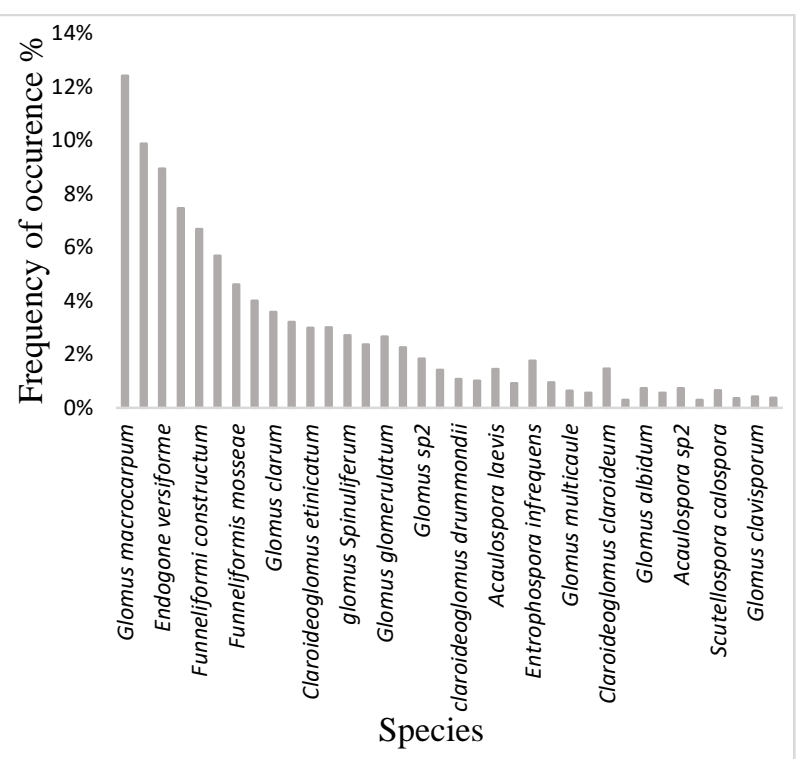

Figure-4: Frequency of occurrence of mycorrhizal species in Zaïo soil

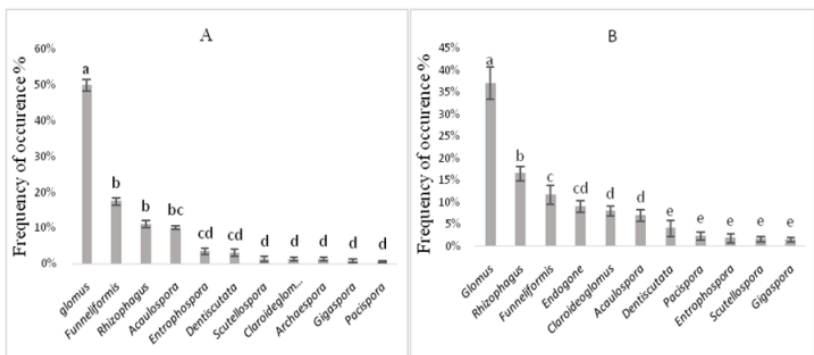

Figure-5: Frequency of occurrence of the genera of endomycorrhizal fungi at both sites: Guercif (A) and Zaïo (B). Data expressed as mean \pm SEM. Values followed by the same lower case letters were not significantly different, whereas values followed by different lower case letters were significantly different $(p<0.05)$

In Guercif soil, the genus Glomus presented a significantly higher frequency of occurrence $(49,78 \%)$ of genera compared to other genus $(p<$ 
0.05), followed by Funneliformis(17.40\%), Rhizophagus

Acaulospora(10,04\%),Entrophospora

$(11,10 \%)$,

Dentiscutata (3\%),Scutellospora

$(3.52 \%)$,

$(1.31 \%)$,

Claroideoglomus (1.29\%), Archaespora (1.29\%), Gigaspora $(0.77 \%)$ and Pasispora (0.51\%). In Zaïo soil, the genus Glomus presented a significantly higher frequency of occurrence $(37.01 \%)$ compared to other genus ( $p<0.05$ ), followed by Rhizophagus (16.47\%), Funneliformis (11.65\%), Endogone (8.95\%), Claroideoglomus (7.90\%), Acaulospora (6.97\%), Dentiscutata (4.01\%), Pacispora (2.26\%), Entrophospora (1.76\%), Scutellospora (1.60\%) and Gigaspora (1.42\%) (Figure 5).

\section{Mycorrhizogenic potential of the studied soils}

All leek plants transplanted into Guercif soil, which was raw, diluted $1 / 4$ or $1 / 16$ were mycorrhizal $(100 \%$ infection). Those transplanted on the highly diluted Guercif soil (1/64 or 1/256) showed an $80 \%$ of mycorrhization (Table 4). On the other hand, the percentage of inoculation of leek plants transplanted into Zaïo soil was decreased with increasing soil dilutions. In fact, at the highest dilution of Zaiio soil $(1 / 256)$, we did not observe any inoculation of leek plants (Table 4).

This result was corroborated by the number of propagules per $100 \mathrm{~g}$ of soil; where Guercif soil presented a high number of mycorrhizal propagules (99 propagules/100 g of soil), whereas Zaiio soil showed a lower number of propagules (34 propagules/100 $\mathrm{g}$ of soil) (Table 5).

Table-4: Number of mycorrhizal plants cultivated on the two soils studied (Guercif and Zaïo)

\begin{tabular}{|c|c|c|c|c|c|c|c|}
\hline \multirow{2}{*}{ Dilutions } & \multirow{6}{*}{ site } & \multicolumn{7}{|c|}{ Rehearsals } & Number of \\
\cline { 3 - 8 } & & $\mathbf{1}$ & $\mathbf{2}$ & $\mathbf{3}$ & $\mathbf{4}$ & $\mathbf{5}$ & $\begin{array}{c}\text { mycorrhizal } \\
\text { plants }\end{array}$ \\
\hline \multirow{2}{*}{$1 / 1$} & Guercif & + & + & + & + & + & 5 \\
\cline { 2 - 8 } & Zaïo & + & + & + & + & + & 5 \\
\hline \multirow{2}{*}{$1 / 4$} & Guercif & + & + & + & + & + & 5 \\
\cline { 2 - 8 } & Zaïo & + & + & + & + & + & 5 \\
\hline \multirow{2}{*}{$1 / 16$} & Guercif & + & + & + & + & + & 5 \\
\cline { 2 - 8 } & Zaïo & + & + & + & + & - & 4 \\
\hline \multirow{2}{*}{$1 / 64$} & Guercif & + & + & + & + & - & 4 \\
\cline { 2 - 7 } & Zaïo & - & - & + & + & - & 2 \\
\hline \multirow{2}{*}{$1 / 256$} & Guercif & + & + & + & + & - & 4 \\
\cline { 2 - 7 } & Zaïo & - & - & - & - & - & 0 \\
\hline
\end{tabular}

The frequency of mycorrhizal leek roots in Guercif soil was significantly higher $(90 \%)$ than that in Zaïo soil (56\%) (Figure 6). Similarly, the intensity of plant root colonization in Guercif soil reached $74 \%$, and was significantly higher than that in Zaïo soil. The latter did not exceeds $31 \%$ after 2 months of planting (Figure 6).

Table-5: MPN estimated for the two soils (Guercif and Zaïo)

\begin{tabular}{|c|c|c|c|}
\hline \multirow{2}{*}{ Site } & \multirow{2}{*}{ MPN } & \multicolumn{2}{|c|}{$\begin{array}{c}\text { Confidence interval at } \\
\text { 95\% (CI 95\%) }\end{array}$} \\
\cline { 3 - 4 } & & Inferior & Superior \\
\hline Guercif & 99.1 & 39.5 & 248.74 \\
\hline Zaïo & 34.04 & 13.56 & 85.44 \\
\hline
\end{tabular}

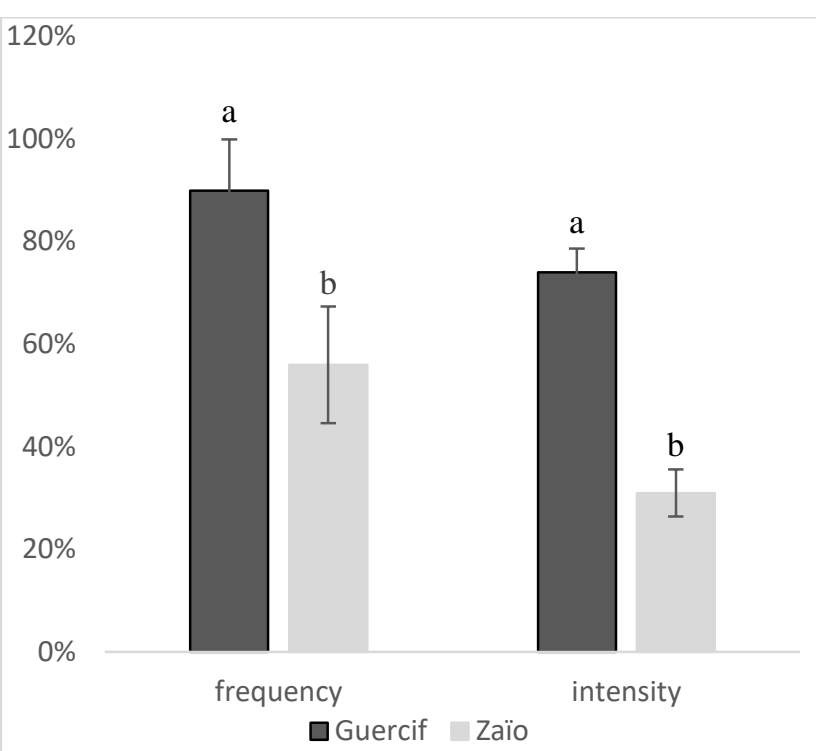

Figure-6: Frequency and intensity of mycorrhization of leek roots after 10 weeks of cultivation. Each of the mycorrhization parameters (frequency and intensity) was treated statistically independently of the other. Data expressed as mean \pm SEM, where values followed by different lowercase letters indicate significant difference using Student's t-test $(p<0.05)$

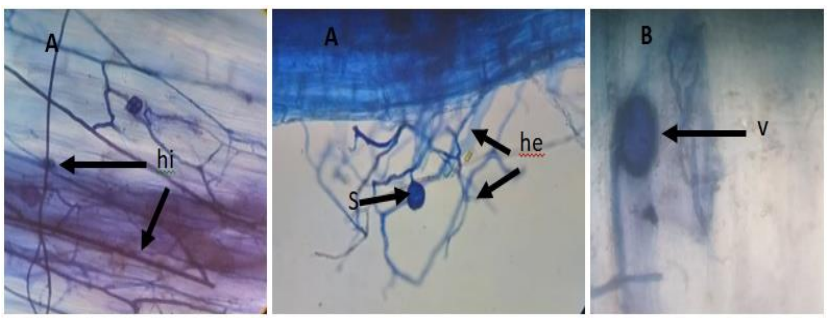

Figure-7: Each site (Guercif (A) Zaïo (B)) developed distinct arbuscular mycorrhizal structure in the leek's roots. V (vesicles), he (extraradicular hyphae), hi (intraradicular hyphae) and S (spores). Samples were observed under $x 400$ magnification

\section{Discussion}


Our results from this study revealed the richness and the diversity of mycorrhizal fungi in two sites in eastern Morocco: Guercif and Zaïo. While the spore density was high at both sites, it was significantly higher in Guercif soil compared to Zaïo soil (386 and 279 spores per $10 \mathrm{~g}$ of soil, respectively). The densities in our study were higher than those reported by different other studies in different areas of Morocco. In fact, previous studies reported spore intensity of 7.8 spores per $10 \mathrm{~g}$ of soil in the rhizosphere of Populus alba in the northwest of Morocco (Talbi et al., 2014) and 6.3 to 9.8 spores per $10 \mathrm{~g}$ of soil in the coastal dunes of Souss-Massa' region of Morocco (Hatimi and Tahrouch, 2007). On the other hand, spore densities from our study are comparable to those obtained in the maize rhizosphere in the agroecological zone of fisheries in Benin (325.9 per $10 \mathrm{~g}$ of soil) (Leslie-Dolorès et al., 2019). However, the spore densities in eastern Morocco in the present study, are noticeably lower than those found in the maize rhizosphere in the food crop agroecological zone south-Borgou in Benin, reaching 1250.15 per $10 \mathrm{~g}$ of soil (Leslie-Dolorès et al., 2019).

The variation in spore densities between different sites may result from micro-climatic variations (Koske, 1987), the physico-chemical and/or microbiological properties of soils (Houngnandan et al., 2009). In fact, Panwar and Tarafdar reported that abiotic factors play an important role in the distribution of mycorrhizal fungi (Panwar and Tarafdar, 2006). In our study, the physico-chemical analyses of the samples of the two soils, indicated that both soils were basic (with a $\mathrm{pH}$ around 8) with low in organic matter content. In addition, we showed that Zaïo soil contains higher levels of phosphorus and nitrogen compared to Guercif soil. This increase in phosphorus and nitrogen in Zaïo soil is probably the consequence of previous intensive use of mineral fertilizers in this site, which contributes to the degradation of the soil microflora and decreases the number of AMF. On the other hand, the higher spore density in Guercif soil could have been the result of the relatively shorter time of exploitation of Guercif's plains, thus the lack of intensive fertilizers uses.

Examination of these two soils showed natural existence of a highly diverse community of fungal isolates of AMF. A total of 57 species of arbuscular mycorrhizal fungi belonging to 11 genera and 5 families were isolated and identified. Guercif soil was found to be richer than Zaïo soil in AMF species (42 species vs 36 species, respectively). Variations in the species composition of arbuscular mycorrhizal fungi were observed between the two sites. These differences could be due to the differences in soil composition and the climate between the two sites, given their different geographical locations.

Since different fungal species have different functional roles, these results are satisfactory, and this increase in species diversity could translate into an increase in functional diversity.

The count of mycorrhizal fungi spores showed a predominance of the genus Glomus, with a frequency of occurrence of $49.78 \%$, represented by 17 species in our samples from Guercif soil. In Zaiio soil, the Glomus frequency, represented by 14 species, was $37.01 \%$. It has been reported that the genus Glomus is the dominant genus in various natural ecosystems (Hijri et al., 2006). This dominance may be associated with the ability of this genus to produce more spores in a shorter time compared to other genera (Bever et al., 1996), as well as its adaptability to drought and soil salinity (Blaszkowski et al., 2002). For instance, Mosse showed that the genus Glomus is often found in neutral or alkaline $\mathrm{pH}$ (Mosse, 1973). In Morocco, the dominance of the genus Glomus has also been observed in the rhizosphere of olive trees in three regions of Morocco (Tafilalt, Zagora and Taounate) (Kachkouch et al., 2014), Citrus (Artib et al., 2016) and in sites adjacent to phosphate mines (El Gabardi et al., 2019).

The mycorrhizogenic potential, (expressed as MPN) was 99 propagules per $100 \mathrm{~g}$ of Guercif soil. This number was lower in Zaïo soil (34 propagules per $100 \mathrm{~g}$ of soil). The estimated values for the two soils in our study remain very high compared to those found in other studies. Azcón-Aguilar et al. reported a value of about 24 propagules /100 $\mathrm{g}$ of soil in open soil (Azcón-Aguilar et al., 2003). However, Meddich et al. reported higher values which reached 1627 propagules per $100 \mathrm{~g}$ in rhizospheric soils of palm groves in tafilalt, Morocco (Meddich et al., 2017).

The mycorrhizal frequency of leek roots transplanted into was significantly higher in Guercif soil $(90 \%)$ compared to that in Zaiio soil $(56 \%)$. This variability in the frequency of mycorrhization from one site to another can be explained by differences in the physico-chemical properties of the soils used. For instance, in contrast to Guercif soil which is low in phosphorus (7.27 ppm), Zaiio soil had 10 times more phosphorus content (74.9 ppm). In fact, our results 
corroborate several other studies suggesting that mycorrhization frequencies are higher in soils with low total phosphorus levels (Kachkouch et al., 2012). Similar to the mycorrhizal frequency, the colonization intensity of plant roots follows the same trend, with higher colonization intensities in Guercif soil $(74 \%)$ compared to that in Zaïo soil (31\%).

The values obtained for the mycorrhizal potential of the soils in our study seem to be correlated with the mycorrhization parameters (frequency and intensity of mycorrhization). It should be noted that the mycorrhizal infectivity potential of a soil depends not only on the number of spores present in the soil, but also on their adaptability and infectivity.

\section{Conclusion}

The analysis of the richness and diversity of mycorrhizal fungi from two sites in the eastern region of Morocco; Guercif and Zaiio showed that these two prospected soils harbor a variety of AMF communities, represented by 57 species, 42 for the Guercif soil and 36 for the Zaïo soil, dominated by the genus Glomus. The Guercif soil had a spore density of 386 spores per $10 \mathrm{~g}$ of soil, a frequency and intensity of root colonization of $90 \%$ and $74 \%$ respectively, as well as a mycorrhizogenic potential of 99 propagules/100 $\mathrm{g}$ of soil which are higher than those found in the Zaiio soil (spore density: 279 spores per $10 \mathrm{~g}$ of soil; Frequency of mycorrhization: 56\%; Mycorrhization intensity: $31 \%$; Mycorrhizogenic potential: 34 propagules/100 g of soil).

This study characterized, the mycorrhizal fungi naturally present in both soils that seem capable of acting as a source of natural inoculum, and have the potential of being a powerful tool in organic farming practices, which are part of sustainable land management.

\section{Acknowledgment}

The authors sincerely thank Labazi Hicham Dr. Research Fellow, Strathclyde Institute of Pharmacy and Biomedical Sciences, for his critical observations, which improved the manuscript.

Disclaimer: None.

Conflict of Interest: None.

Source of Funding: This study was financially supported by Ministry of Higher Education and
Scientific Research, Morocco.

\section{References}

Adesemoye OA and Kloepper JW, 2009. Plantmicrobes interactions in enhanced fertilizer-use efficiency. Appl. Microbiol. Biotechnol. 85(1): 112.

Alexander M, 1965. Most probable number method for microbial populations, pp. 1467-1472. Dans Methods of soil analysis. Part 2. Chemical and microbiological properties. C A Black American Society of Agronomy, Madison, Wisconsin, USA.

Artib M, Chliyeh M, Touati J, Talbi Z, Selmaoui K, Ouazzani Touhami A, Benkirane R and Douira A, 2016. Study of Arbuscular mycorrhizal fungi diversity in the rhizosphere of citrus grown in Morocco. IJAPBC. 5(3): 2277-4688.

Azcón-Aguilar C, Palenzuela J, Roldan A, Bautista S, Vallejo R and Barea JM, 2003. Analysis of the mycorrhizal potential in the rhizosphere of representative plant species from desertificationthreatened Mediterranean shrublands. Appl. Soil Ecol. 22(1): 29-37.

Begum N, Qin C, Ahanger MA, Raza S, Khan MI, Ashraf M, Ahmed N and Zhang L, 2019. Role of Arbuscular Mycorrhizal Fungi in Plant Growth Regulation: Implications in Abiotic Stress Tolerance. Front. Plant Sci. 10: 1068.

Bencherif K, Boutekrabt A, Fontaine J, Laruelle F, Dalpè Y and Sahraoui AL, 2015. Impact of soil salinity on arbuscular mycorrhizal fungi biodiversity and microflora biomass associated with Tamarix articulata Vahll rhizosphere in arid and semi-arid Algerian areas. Sci. Total Environ. 533: 488-94.

Bever JD, Westover KM and Antonovics J, 1996. Incorporating the soil community into plant population dynamics: the utility of the feedback approach. J. Ecol. 85: 561-763.

Blaszkowski J, Tadych M and Madej T, 2002. Arbuscular mycorrhizal fungi (Glomales, Zygomycota) of the bledowska desert, Poland. Acta Soc. Bot. Pol. 71(1): 71-85.

Cochran WG, 1950. Estimation of bacterial densities by means of the "most probable number". Biometrics. 6(2): 105-116.

Damon J, 2003. Les prévisions démographiques de l'ONU à l'horizon 2050. RPSF. 74(1): 88-95.

Duponnois R, Ramanankierana H, Hafidi M, 
Baohanta R, Baudoin E, Thioulouse J and Lebrun M, 2013. Des ressources végétales endémiques pour optimiser durablement les opérations de réhabilitation du couvert forestier en milieu méditerranéen et tropical: exemple des plantes facilitatrices vectrices de propagation des champignons mycorhiziens. Comptes Rendus Biol. 336(5-6): 265-272.

El Gabardi S, Chliyeh M, Selmaoui K, Ouazzani Touhami A, El Modafar C, Abdelkarim Filali Maltouf A, Elabed S, Ibnsouda Koraichi S, Amir S, Moukhli A, Benkirane R and Douira A, 2019. Study of the endomycorrhizogenic potential of phosphate laundered sludge. Wulfenia. 26(5): 3857.

Fisher RA and Yates F, 1970. Statistical Tables for Biological Agriculture and Medical Research, 6th ed. Davien Hafner Publication Company, New York, USA.

Gerdemann JW and Nicolson TH, 1963. Spores of mycorrhizal Endogone species extracted from soil by wet sieving and decanting. Transact. Brit. Mycol. Soc. 46(2): 235-244.

Gianinazzi-Pearson V, Gianinazzi S and Trouvelot A, 1985. Evaluation of the infectivity and effectiveness of indigenous vesicular-arbuscular fungal populations in some agriculturable soils in Burgundy. Canad. J. Bot. 63: 1521-1524.

Giovannetti M, Avio L and Salutini L, 1991. Morphological, cytochemical, and ontogenetic characteritics of a new species of vesiculararbuscular mycorrhizal fungus. Canad J. Bot. 69(1): 161-167.

Hatimi A and Tahrouch S, 2007. Caractérisations chimique, botanique et microbiologique du sol des dunes littorales du Souss- Massa. J. Biomatec. Echo. 2(5): 85-97.

Hijri I, Sýkorová Z, Oehl F, Ineichen K, Mäder P, Wiemken A and Redecker D, 2006. Communities of arbuscular mycorrhizal fungi in arable soils are not necessarily low in diversity. J. Mol. Ecol. 15(8): 2277-89.

Houngnandan P, Yemadje RG, Kane A, Boeckx P and Van Cleemput O, 2009.Indigenous Glomales of Claire forest Isoberlinia doka (Craib and Stapf) in Wari-Maro in central Benin. Tropicultura. 27(2): 83-87.

INVAM, 2017. Species Descriptions from Reference Cultures. International Culture Collection of (Vesicular) Arbuscular Mycorrhizal Fungi, West Virginia University. URL: http://fungi.invam.wvu.edu/the-fungi/speciesdescriptions.html.

Jung SC, Martinez-Medina A, Lopez-Raez JA and Pozo MJ, 2012. Mycorrhiza-induced resistance and priming of plant defenses. J. Chem. Ecol. 38(6): 651-64.

Koske RE, 1987. Distribution of VA mycorrhizal fungi along a latitudinal temperature gradient. Mycologia. 79(1): 55-68.

Kachkouch W, Touati J, Ouazzani Touhami A, FilaliMaltouf A, El Modafar C, Moukhli A, Oukabli A, Benkirane R and Douira A, 2014. Diversity of arbuscular mycorrhizal fungi in the rhizosphere of Olea europaea in three regions of Morocco (Tafilalt, Zagora and Taounate). Int. J. Pure Appl. Biosci. 2(5): 178-195.

Kachkouch W, Ouazzani Touhami A, Filali Maltouf A, El Modafar C, Moukhli A, Oukabli A, Benkirane R and Douira A, 2012. Arbuscular mycorrhizal fungi species associated with rhizosphere of Olea europaea L. in Morocco. J. Anim. Plant Sci. 15(3): 2275-2287.

Leslie-Dolorès RB, Howell BH, Appolinaire A and Charlotte ZP, 2019. Diversité des champignons mycorhiziens arbusculaires associés à la culture du maïs (Zea mays L.) au Bénin. Int. J. Biol. Chem. Sci. 13(2): 597-609.

Meddich A, Ait El Mokhtar M, Wahbi S and Boumezzough A, 2017. Evaluation of the mycorrhizal potential in relation with the physico-chemical properties of soils in Moroccan palm groves (Marrakech and Tafilalet). Cahiers Agric. 26(4): 45012.

Morton JB and Benny J, 1990. Revised classification of arbuscular mycorrhizal fungi (Zygomycetes): a new order, Glomales, two new suborders, Glominae and Gigasporinae, and two new families, Acaulosporaceae and Gigasporaceae, with an amendation of Glomaceae. Mycotaxon. 37: 471-491.

Morton JB and Bentivenga SP, 1994. Levels of diversity in endomycorrhizal fungi (Glomales, Zygomycetes) and their role in defining taxonomic and nontaxonomic groups. Plant Soil. 159: 47-59.

Mosse B, 1973. Advances in the study of vesiculararbuscular mycorrhiza. Annu. Rev. Phytopathol. 11(1): 171-196.

Pamiske M, 2008. Arbuscular mycorrhiza: the mother of plant root endosymbioses. Nat. Rev. Microbiol. 6(10): 763-775. 
Panwar J and Tarafdar JC, 2006. Distribution of three endangered medicinal plant species and their colonization with arbuscular fungi. J. Arid. Environ. 65(65): 337-350.

Phillips JM and Hayman DS, 1970. Improved procedures for clearing roots and staining parasitic and vesicular-arbuscular fungi for rapid assessment of infection. Transact. Brit. Mycol. Soc. 55(1): 158-161.

Plenchette C, Perrin R and Duver P, 1989. The concept of soil infectivity and a method for its determination as applied to endomycorrhizas. Canad. J. Bot. 67(1): 112-115.

Plenchette C, Clermont-Dauphin C, Meynard JM and Fortin JA, 2005. Managing arbuscular mycorrhizal fungi in cropping systems. Canad. J. Plant Sci. 85(1): 31-40.

Porter WM, 1979. The 'most probable number' method for enumerating infective propagules of vesicular arbuscular mycorrhizal fungi in soil. Aust. J. Soil Res. 17(3): 515-519.

Rillig MC, Wright SF and Eviner VT, 2002. The role of arbuscular mycorrhizal fungi and glomalin in soil aggregation: comparing effects of five plants species. Plant Soil. 238(2): 325-333.

Sally ES, Andrew SF and Iver JI, 2003. Mycorrhizal Fungi Can Dominate Phosphate Supply to Plants Irrespective of Growth Responses. Plant Physiol. 133(1): 16-20.

Schenk NC and Perez Y, 1990. Manual for the identification of VA mycorrhizal fungi. Univ of Florida Press, Florida, USA.

Schenk NC and Perez Y, 1987. Manual for the identification of VA Mycorrhizal fungi. First Edition INVAM. Univ of Florida Press, Florida,
USA.

Talbi Z, Chliyeh M, Selmaoui K, Ouazzani Touhami A, Benkirane R and Douira Allal, 2014. Mycorrhizal Status Juncus maritimus, Riparian Sepecies of Sidi Boghaba Reserve (Northwest of Morocco. Int. J. Recent Sci. Res. 5(4): 792-795.

Trouvelot A, Kough $\mathrm{J}$ and Gianinazzi-Pearson V, 1986. Evaluation of VA infection levels in root systems. Research for estimation methods having a functional significance, pp. 217-221. Dans Gianinazzi-Pearson V et Gianinazzi S, Physiological and Genetical Aspects of Mycorrhizae. INRA Press, Paris, France.

Walker C and Mize C, 1982. Population of endogonaceous fungi at two locations in central Iowa, 1982. Canad. J. Bot. 60(2): 2518-2529.

Wilson JM and Trinick MJ, 1982. Factors affecting the estimation of numbers of infective propagules of vesicular arbuscular mycorrhizal fungi by the most probable number method. Aust. J. Soil Res. 21: 73-81.

\section{Contribution of Authors}

Chafai W: Conceived idea, designed research methodology, conducted experiments, data analysis, literature review and article write up EL Gabardi S: Conducted experiments and collected data

Douira A: Literature review, article editing and write up

Khalid A: Conceived idea, final editing and approval of article 